\title{
Open Subpectoral Biceps Tenodesis versus Arthroscopic Repair for SLAP Tears in Patients under 30 (224)
}

Christopher Colasanti, MD ${ }^{1}$, Eoghan Hurley, MB, BCh, BAO, $\mathrm{MCh}^{2}$, Nathan Lorentz, Kirk Campbell, MD ${ }^{1}$, Michael Alaia, MD², Eric Strauss, $\mathrm{MD}^{4}$, Laith Jazrawi, $\mathrm{MD}^{5}$, Bogdan Matache

NYU Langone Orthopedic Hospital ${ }^{1}$ NYU Langone ${ }^{2}$ NYU Langone Health ${ }^{3}$ NYU Langone Orthopedics ${ }^{4}$ NYU Hospital for Joint Diseases $^{5}$

Objectives: The purpose of this study is to compare the outcomes of mini-open subpectoral biceps tenodesis (BT) to arthroscopic repair (AR) for SLAP tears in patients under 30.

Methods: A retrospective review of patients who underwent either isolated BT or AR for the diagnosis of a SLAP tear was performed. Patients with a follow-up duration of $<24$ months were excluded. The American Shoulder \& Elbow Surgeons (ASES) score, Visual Analogue Scale (VAS), Subjective Shoulder Value (SSV), patient satisfaction, willingness to undergo surgery again, revisions, and return to work/sport were evaluated. A p value of $<0.05$ was considered to be statistically significant.

Results: Our study included 103 patients in total; 29 patients were treated with BT, and 74 were treated with AR. The mean age was 24.8 years, $79.4 \%$ were male, and the mean follow-up duration was 60 months. At final follow up, there was no difference between treatment groups in any of the functional outcome measures assessed $(\mathrm{p}>0.05)$. Overall, there was no significant difference in the total rate of RTP (BT: $76.3 \%$, AR: 85\%; $\mathrm{p}=0.53$ ), timing of RTP (BT: 8.8 months, AR: 9.4 months; $\mathrm{p}=0.61$ ), and total rate of RTP among overhead athletes (BT: $84.2 \%$, AR: $83.3 \% ; \mathrm{p}=1$ ). However, there was a significantly lower rate of revision surgery with BT $(0 \%)$ as compared to $\operatorname{AR}(14.1 \% ; p=0.03)$.

Conclusions: In patients under the age of 30 with isolated SLAP tear pathology, BT is a reliable alternative to AR, with a low rate of revision surgery, and excellent patient reported outcomes.

The Orthopaedic Journal of Sports Medicine, 9(10)(suppl 5)

DOI: $10.1177 / 2325967121500332$

(C) The Author(s) 2021

This open-access article is published and distributed under the Creative Commons Attribution - NonCommercial - No Derivatives License (https://creativecommons.org/licenses/by-nc-nd/4.0/), which permits the noncommercial use, distribution, and reproduction of the article in any medium, provided the original author and source are credited. You may not alter, transform, or build upon this article without the permission of the Author(s). For article reuse guidelines, please visit SAGE's website at http://www.sagepub.com/journals-permissions. 